\title{
Théorie de la diffraction de photoélectrons appliquée au dichroïsme circulaire et à l'émission de photoélectrons polarisés en spin
}

\author{
M.A. Van Hove* et C.S. Fadley*** \\ * Lawrence Berkeley National Laboratory, University of California, Berkeley, CA 94720, U.S.A. \\ ** Department of Physics, University of California-Davis, Davis, CA 95616, U.S.A.
}

\begin{abstract}
Résumé : Nous esquissons des méthodes théoriques pour calculer le dichroïsme circulaire (DC), la polarisation en spin (PS), le dichroïsme magnétique circulaire (DMC), et le dichroïsme magnétique linéaire (DML), phénomènes qui existent en photoémission de niveaux de coeur excitée par de la lumière polarisée circulairement et linéairement. Le présent formalisme généralise nos logiciels antérieurs qui calculent la multidiffraction des photoélectrons jusqu'à convergence complète. Les résultats de ces nouveaux calculs sont comparés à des données expérimentales obtenues par plusieurs groupes pour plusieurs cas illustratifs : le DC en photoémission de surfaces "non-chirales" [CO sur Pd(111); Si(001)] ; la PS en photoémission d'atomes nonmagnétiques et magnétiques, et de surfaces [Xe ; W(110); $\mathrm{Fe}(110)]$; et le DMC en photoémission de $\mathrm{Fe}(110)$. Nous discutons les conditions qui engendrent le dichrö̈sme circulaire en l'absence de chiralité structurelle ou de magnétisme. Nous mettons également l'accent sur le rapport étroit entre le DC non-magnétique et le DC magnétique, ainsi que sur le comportement très anisotrope de la PS et son importance dans les études de l'ordre magnétique par diffraction de photoélectrons polarisés en spin.
\end{abstract}

\section{INTRODUCTION}

La disponibilité de rayonnement synchrotron de haute intensité avec polarisation variable entre circulaire et linéaire crô̂t constamment, grâce à la mise en service en différents pays du monde de nouveaux synchrotrons de la troisième génération et de nouvelles lignes de lumière. Ceci a mené à plusieurs études récentes de dichroïsme circulaire et linéaire dans l'excitation de photoélectrons à partir de niveaux atomiques de coeur, en particulier pour des surfaces de monocristaux. De plus, la polarisation en spin des photoélectrons émis peut être étudiée plus facilement avec ces nouveaux instruments. Le dichroïsme et la polarisation en spin ont tous deux une grande importance pour l'étude de l'état magnétique d'une surface. Mais il faut d'abord apprendre à distinguer les effets magnétiques des effets non-magnétiques. Nos recherches décrites ici examinent en partie cette question fondamentale.

Par définition, le dichroïsme circulaire mesure l'asymétrie due au renversement de la polarisation de la lumière ; on obtient cette asymétrie $A_{C D}$ en faisant le rapport de la différence sur la somme de deux intensités, à savoir des intensités de photoémission obtenues avec polarisation droite et gauche, respectivement. Le dichrö̈sme linéaire est défini de façon semblable, mais s'applique aú càs magnétique et s'obtient en contrastant les intensités de photoémission avant et après rotation de $180^{\circ}$ de la magnétisation de l'échantillon.

La polarisation en spin peut se mesurer avec des détecteurs qui distinguent le sens du spin de l'électron $(+1 / 2$ ou $-1 / 2)$. Comme ces détecteurs sont peu efficaces, on a de préférence recours à une méthode alternative, disponible lorsque les photoélectrons sont émis à des énergies suffisamment séparées par l'interaction spin-orbite. Il suffit alors de mesurer individuellement les intensités émises à ces énergies différentes pour obtenir la polarisation en spin. Cette polarisation est définie de la même façon que l'asymétrie dichroïque, à savoir comme le rapport de la différence sur la somme des intensités de photoémission obtenues avec spin $+1 / 2$ et $-1 / 2$, respectivement.

Le dichroïsme peut être détecté le plus facilement en mesurant le coefficient d'absorption de rayons X. Cette approche donne lieu par exemple au "dichroïsme magnétique circulaire" (DMC ; soit en anglais MCD ou "magnetic circular dichroism"), ou aussi à la "structure fine dans l'absorption de rayons X avec polarisation en spin" (en anglais SPEXAFS ou "spin polarized extended $x$-ray absorption fine structure"). 
On peut également examiner la diffraction plus en détail par le moyen des spectres en énergie et des distributions en fonction d'angles de sortie des photoélectrons émis à partir d'un niveau de coeur. Ceci mène, par exemple, au "dichroïsme circulaire des distributions en angle de photoélectrons" (DCDA ; soit en anglais CDAD ou "circular dichroism in photoelectron angular distribution") ou à la "diffraction polarisée en spin de photoélectrons" (DPSP ; soit en anglais SPPD ou "spin-polarized photoelectron diffraction"). Donc, cette famille de techniques offre de nouvelles méthodes flexibles pour étudier l'ordre structural et magnétique à courte distance autour d'un type donné d'atome absorbant.

Nous présentons dans cet article nos calculs théoriques de l'émission de photoélectrons dans la région proche d'une surface, traitant en particulier l'excitation d'un niveau de coeur séparé en plusieurs sousniveaux par l'intéraction spin-orbite. Cette émission est obtenue à l'aide de rayonnement à polarisation variable. Les calculs comprennent tous les processus de diffusion (diffraction) pendant l'émission des photoélectrons jusqu'à convergence complète (une dizaine de diffusions peuvent être nécessaires). Les calculs permettent également d'obtenir le dichroïsme circulaire (DC) et la polarisation en spin (PS) résultant de l'émission de photoélectrons. Ces résultats sont comparés à des données expérimentales aussi bien pour des systèmes non-magnétiques que magnétiques, et leurs implications sont discutées pour les études de photoémission et d'absorption de rayons X.

Notre formalisme théorique commence avec la méthode de la fonction de Green séparable de Rehr et Albers [1], telle qu'elle a été réalisée dans un algorithme à base de multi-diffraction dans un groupe de 2 à quelques centaines d'atomes, développé par Kaduwela, Friedman et Fadley [2]. Nous avons montré précédemment que cette méthode s'applique très bien à l'excitation de photoélectrons à partir de lumière non-polarisée ou polarisée linéairement. La polarisation circulaire est représentée simplement par des opérateurs $\hat{\mathbf{x}} \pm i \hat{\mathbf{y}}$ (pour la propagation de la lumière dans la direction $z$ ), au lieu d'opérateurs $\hat{\mathbf{x}}$ ou $\hat{\mathbf{y}}$ qui décrivent le cas polarisé linéairement ; ceci permet par conséquent les transitions $m_{j} \rightarrow m_{j} \neq 1$. Les effets magnétiques sont représentés respectivement par l'inclusion ou l'exclusion de l'énergie d'échange dans la diffusion de l'électron par un atome, suivant le sens relatif du spin électronique par rapport à la direction du moment magnétique de l'atome. Le logiciel permet toutes les orientations relatives de la lumière incidente, des axes cristallins, de la magnétisation, de l'émission, et du spin de l'électron détecté. Des détails supplémentaires sont publiés ailleurs [3].

\section{DC EN PHOTOÉMISSION ANGULAIRE ÉMANANT DE CO "NON-CHIRAL" SUR Pd(111)}

Comme première application de ce formalisme, nous avons effectué des calculs de dichrö̈me circulaire pour simuler une expérience impliquant des molécules de CO adsorbées sur une surface de Pd(111), expérience réalisée par Bansmann et al. [4]. La surface fut illuminée par du rayonnement synchrotron à polarisation circulaire droite et gauche, avec un angle d'incidence de $50^{\circ}$ par rapport à la normale à la surface. Des photoélectrons de coeur émis du niveau $C$ 1s furent détectés le long d'un arc qui inclut la direction d'émission normale.

Sauf pour l'émission dans la direction normale, ce système a une asymétrie chirale due à la polarisation de la lumière, même si les positions atomiques sont symétriques par rapport au plan contenant la normale à la surface et la direction de la lumière incidente. Par conséquent, on s'attend à une différence entre les intensités d'émission dues à la lumière polarisée circulairement à droite ou à gauche, et on observe donc de façon générale un dichrö̈sme non nul (sauf pour l'émission dans le plan de symétrie mentionné cidessus). Ce dichroïsme, dépendant de l'angle, fut mesuré pour une série d'énergies cinétiques des électrons dans la gamme de 22 à $97 \mathrm{eV}$, et l'on observe des effets prononcés allant jusqu'à $50 \%$ ou même $75 \%$ d'asymmétrie.

Nos calculs [5] reproduisent bien les données expérimentales sur les gammes complètes d'angles et d'énergies, et correspondent également bien aux calculs pour une molécule isolée (c'est à dire sans substrat) effectués par McKoy et Stephens avec un modèle théorique très différent [6]. Ceci indique une influence relativement faible des positions des molécules de $\mathrm{CO}$ par rapport au substrat métallique sousjacent, due à la distance relativement grande entre molécule et métal. Néanmoins, les calculs prédisent une sensibilité suffisante à ces paramètres géométriques pour s'attendre à pouvoir déterminer la géométrie d'adsorption moléculaire à partir d'expériences soigneuses et étendues de ce type ; ceci peut par exemple se faire en produisant des courbes calculées pour plusieurs géométries d'adsorption et en choisissant celle qui correspond le mieux à l'expérience. 


\section{EXPERIMENT}
(a) LCP
(b) RCP
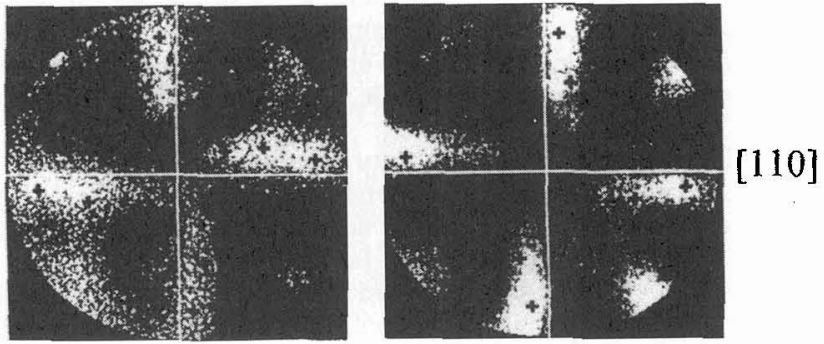

\section{THEORY}

(c) LCP

(d) RCP
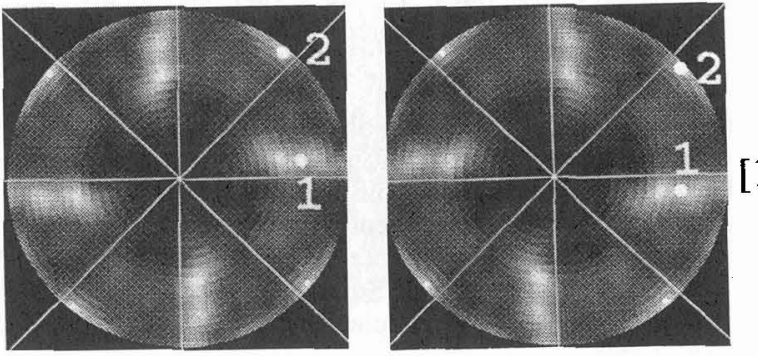

$[110]$

(e) Unpolarized

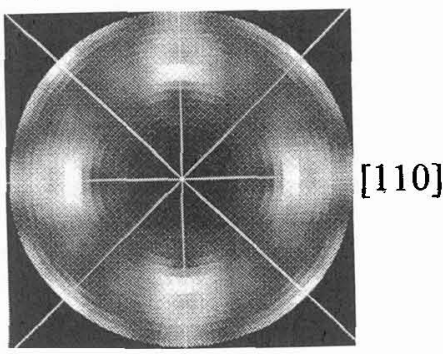

Figure 1. (a), (b) : Distributions angulaires de la diffraction de photoélectrons pour l'émission du niveau Si $2 \mathrm{p}$ de $\mathrm{Si}(001)$, à une énergie cinétique des électrons de $250 \mathrm{eV}$, observées par Daimon et al. [7]. Le centre de chaque distribution correspond à l'émission normale, tandis que les bords circulaires sont à environ $45^{\circ}$. de la direction normale. Les distributions (a) et (b) ont été obtenues avec de la lumjère à polarisation circulaire gauche et droite, respectivement. Les croix indiquent les positions déviées des pics tels qu'ils sont prédits par le modèle de focalisation de Daimon et al. Les chiffres 1 et 2 illustrent la correspondance de pies déviés dans les deux sens. (c), (d) : Les distributions correspondantes calculées, comprenant la multi-diffraction et l'interférence complète dans l'état final entre les canaux $s$ et $\mathbf{A}$. (e): Résultat calculé pour la lumière non-polarisée, qui peut être obtenu en moyennant les distributions (c) et (d). 


\section{DC EN PHOTOÉMISSION ÉMANANT DE Si(001) "NON-CHIRAL"}

Le dichroïsme circulaire en photoémission a été mesuré aussi pour la surface nue d'un monocristal nonmagnétique de Si(001) par Daimon et al. [7]. L'expérience a utilisé les émissions $2 \mathrm{~s}$ et $2 \mathrm{p}$ du Si avec différentes énergies cinétiques des électrons dans la gamme de 150 à $450 \mathrm{eV}$. Elle a mesuré, pour chaque énergie, les distributions bidimensionelles d'intensités émises pour tous azimuts sur une gamme de l'angle polaire d'environ 0 à $45^{\circ}$ (mesuré à partir de la normale à la surface) : voir les figures 1(a) et (b).

Bien que la direction d'incidence de la lumière soit normale à la surface et que la structure de la surface soit non-chirale (elle a des plans de symétrie perpendiculaires à la surface), on observe une chiralité prononcée dans les données mesurées ; la chiralité se manifeste le plus clairement sous la forme de "rotations azimutales" des pics dominants dans la distribution en angle de la diffraction des photoélectrons. Les figures 1(a) et (b) en particulier montrent de tels pics d'intensité tournés dans le sens des aiguilles d'une montre comme dans le sens contraire, respectivement, pour la lumière incidente polarisée droite et gauche. La lumière non-polarisée produirait des pics dans les directions moyennées, donc symétriques, ainsi que le suggère notre calcul illustré dans la figure 1(e).

Les résultats de nos calculs de DC pour ce cas de Si(001), illustrés dans les figures 1(c) et (d), reproduisent bien les observations expérimentales. En particulier, les calculs confirment que des rotations de pics sont attendues de façon générale avec la lumière polarisée circulairement, y compris pour le cas de surfaces non-chirales.

Pour expliquer de façon simple l'origine de la rotation observée des pics, Daimon et al. [7] ont proposé un modèle approximatif très utile, basé sur la focalisation vers l'avant. Ce phénomène de focalisation en avant s'explique par la tendance des électrons à être diffusés par un atome de préférence dans la direction en avant, c'est à dire avec une déviation relativement faible de la direction de l'atome émetteur vers l'atome diffuseur ; ceci se produit surtout pour des énergies au-dessus d'environ $200 \mathrm{eV}$. Chaque atome diffuseur agit ainsi comme une lentille convergente. Suivant ce modèle, les pics majeurs dans la figure 1 représentent donc toutes les directions reliant un atome émetteur quelconque à des atomes proches qui jouent le rôle de focaliseurs (lentilles).

Pour la lumière non-polarisée ou polarisée linéairement, cette focalisation vers l'avant est concentrée sur l'axe interatomique, révélant donc directement les directions interatomiques. Cependant, si la lumière a une polarisation circulaire, l'onde électronique qui heurte un atome diffuseur possède une valeur non-nulle du nombre quantique magnétique $\mathrm{m}$, imposée par la règle de sélection dipolaire. Ceci produit une rotation des fronts d'onde d'un angle de l'ordre de 5 à $15^{\circ}$, dans le sens des aiguilles d'une montre ou dans le sens opposé, suivant le sens de polarisation de la lumière. La focalisation en avant est alors aussi déviée d'un angle semblable, comme Daimon et al. ont observé expérimentalement d'abord et calculé théoriquement ensuite. Cependant, pour prédire quantitativement l'angle de rotation de chaque pic, nous avons montré qu'il faut calculer la multi-diffraction complète, tout en évaluant les amplitudes exactes des différentes composantes des ondes sphériques impliquées dans tout événement de diffusion.

\section{PHOTOÉMISSION POLARISÉE EN SPIN ÉMANANT DE Xe ATOMIQUE ET DE W(110) NON-MAGNÉTIQUE}

Nous avons calculé, pour deux cas, la polarisation en spin (PS) qui résulte de la séparation des niveaux de coeur : l'effet Fano. Cet effet est dû au fait que les différentes transitions entre niveaux électroniques donnent lieu à des polarisations en spin différentes, qui ressoretent clairement dans le cas de niveaux de coeur séparés. Nous avons comparé nos résultats de ce type avec deux séries d'expériences : le cas de photoémission $5 \mathrm{p} \rightarrow \mathrm{s}+\mathrm{d}$ pour le $\mathrm{Xe}$ atomique et le cas de la photoémission $4 \mathrm{f} \rightarrow \mathrm{d}+\mathrm{g}$ pour la surface W(110) non-magnétique.

Dans le cas du Xe atomique, Heckenkamp et al. [8] ont mesuré la PS du niveau 5p1/2 en fonction de l'angle de diffusion à une énergie cinétique électronique très faible de $1,1 \mathrm{eV}$. Nos calculs reproduisent très bien les données, vérifiant en particulier la méthode théorique utilisée ici, du moins pour ce cas relativement simple. 

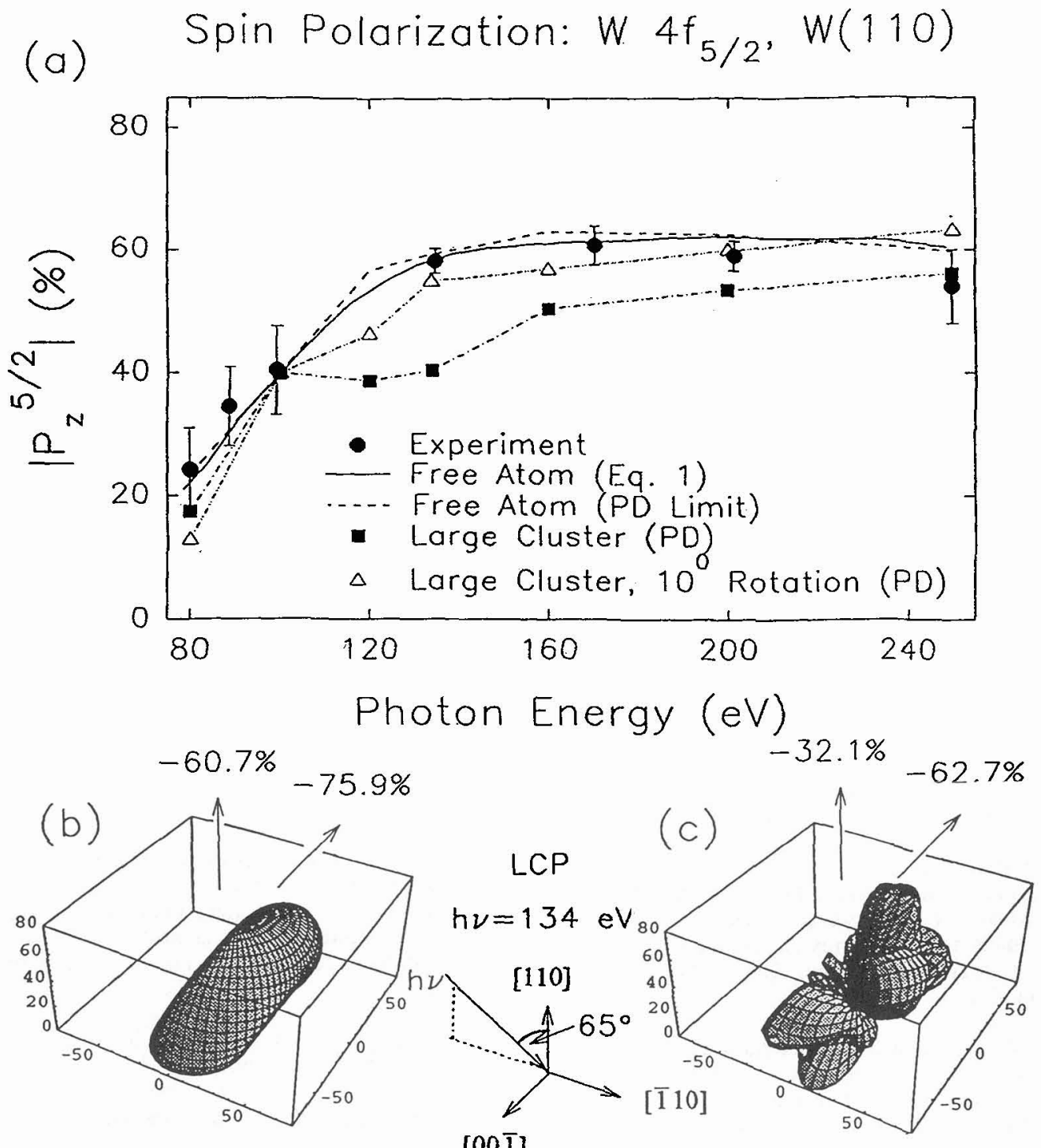

$[00 \overline{1}]$

Figure 2. (a): Dépendance en fonction de l'énergie photonique de la polarisation en spin du pic de photoémission $\mathrm{W} 4 \mathrm{f}_{5 / 2}$ excité par la lumière polarisée circulairement pour la surface W(110). Les données expérimentales (cercles pleins) sont comparées à plusieurs résultats calculés : pour un atome unique (courbe continue); pour le même cas, mais en utilisant un programme de diffraction de photoélectrons ignorant les atomes avoisipants (courbe en tirets); pour un grand groupe d'atomes avec l'orientation nominale expérimentale, à l'aide d'un calcul avec multi-diffraction (courbe à carrés pleins) ; et aussi pour le même groupe d'atomes pivoté de $10^{\circ}$ en azimut (courbe à triangles). (b) : Représentation en trois dimensions de la polarisation du niveau $W 4 f_{5 / 2}$ pour une énergie d'excitation de $134 \mathrm{eV}$ en fonction de la direction d'émission de l'électron pour un atome isolé. (c) : Comme (b), mais pour un groupe de 5 atomes de $W$ avec multi-diffraction. 
Dans le cas de la surface W(110), Starke et al. [9] ont étudié la dépendance énergétique de la PS pour la photoémission d'un solide non-magnétique ; ils ont utilisé une géométrie de diffraction fixe, et une gamme d'énergie cinétique beaucoup plus élevée, allant de 80 à $240 \mathrm{eV}$. Les bandes de photoémission dues à la séparation spin-orbite des niveaux W $4 \mathrm{f} 7 / 2$ et $4 \mathrm{f} 5 / 2$ sont bien résolues et montrent de fortes polarisations, avec des signes opposés le long de l'axe de propagation de la lumière : environ $-35 \%$ et $+50 \%$, respectivement. Le rapport de ces polarisations $(-1,4 \pm 0,1)$ est très proche de la valeur théorique attendue pour des atomes de $\mathrm{W}$ isolés [10] ; nos calculs montrent que cette proportion reste valable aussi pour l'état solide, même avec l'inclusion de diffusion simple ou multiple par les atomes voisins [9].

La figure 2(a) montre la dépendance énergétique de la PS mesurée pour le niveau 4f5/2, comparée avec des calculs pour plusieurs cas différents. Le premier cas est l'atome libre, soit considéré comme atome isolé [10], soit calculé avec notre logiciel multi-atome en l'absence de diffuseurs avoisinants. L'autre cas est un grand groupe d'atomes représentant un segment de surface ; nous avons donné deux orientations azimutales à ce segment : d'une part l'orientation expérimentale nominale, et d'autre part une orientation azimutale tournée de $10^{\circ}$ par rapport à la première (ceci pour examiner la possibilité d'une incertitude expérimentale dans l'orientation azimutale de l'échantillon). On trouve une bonne correspondance entre les calculs et l'expérience, surtout pour le groupe d'atomes pivoté de $10^{\circ}$ par rapport à la géométrie expérimentale nominale. La figure 2(b) illustre la dépendance angulaire de l'émission calculée pour la polarisation venant du niveau W $4 \mathrm{f5} / 2$ à $134 \mathrm{eV}$ pour un atome isolé [un des points dans la figure 2(b)] ; la figure 2(c) donne le résultat correspondant pour un groupe de 5 atomes (un émetteur dans la deuxième couche atomique de la surface et 4 diffuseurs dans la couche extérieure de la surface). Ces résultats illustrent en plus l'effet important que peut avoir la diffusion simple ou multiple des électrons par les atomes avoisinants sur la polarisation de l'électron émis dans l'état final.

\section{DICHROÏSME MAGNÉTIQUE CIRCULAIRE ET POLARISATION EN SPIN POUR LA PHOTOÉMISSION ÉMANANT DE Fe(110) FERROMAGNÉTIQUE}

Finalement, nous présentons nos calculs pour le dichroïsme magnétique circulaire (DMC) et la photoémission polarisée en spin (PS) de $\mathrm{Fe}(110)$. Nous les comparons avec deux ensembles différents de résultats expérimentaux disponibles pour ce système $[11,12]$.

Baumgarten et al. [11] ont mesuré l'intensité de photoélectrons émis à partir des niveaux Fe 2p1/2 et $2 \mathrm{p} 3 / 2$ venant d'une surface $\mathrm{Fe}(110)$, magnétisée suivant son axe préférentiel, en utilisant de la lumière à polarisation circulaire gauche. Les mêmes mesures ont été faites ensuite après l'inversion de la direction de magnétisation ; ceci revient de façon équivalente à mesurer l'intensité du premier système avec une polarisation circulaire droite. Une tentative pour simuler théoriquement ces résultats a aussi été faite par Ebert et al. [13].

La figure 3 compare nos résultats avec les calculs d'Ebert et al. [13] pour la lumière à polarisation circulaire gauche, et aussi avec les résultats expérimentaux de Baumgarten et al. [11]. La figure 3(a) montre en courbes en tirets les spectres pour les niveaux mj individuels calculés suivant la méthode d'Ebert et al. [13] ; la courbe continue représente le spectre total, somme des spectres individuels. Les barres verticales indiquent nos intensités calculées pour chaque niveau mj, ces niveaux étant séparés d'un intervalle déterminé de façon empirique comme l'ont fait également Ebert et al. [13] ; ceci permet de représenter approximativement les séparations des multiplets dans l'état final. La figure indique également les degrés de polarisation calculés pour chaque niveau. Nos calculs ont été réalisés pour un groupe de 5 atomes de $\mathrm{Fe}$ avec multi-diffraction complète.

Les courbes en tirets de la figure 3(b) montrent les spectres correspondants à nos niveaux $m_{j}$ pour la lumière à polarisation circulaire gauche, élargis pour correspondre aux largeurs des pics expérimentaux. La somme de ces contributions produit alors le spectre total pour la polarisation circulaire gauche, représenté par la courbe supérieure en tirets, qui coüncide à peu près avec la courbe continue. Cette courbe continue correspond à notre spectre total pour la lumière à polarisation circulaire droite, calculé de la même manière. Pour permettre la comparaison, le spectre total obtenu par Baumgarten et al. en polarisation linéaire [11] est représenté par la courbe en pointillés dans la figure 3(b). Nous avons ajouté un fond continu à notre spectre total pour permettre une comparaison plus réaliste avec l'expérience ; ce fond continu a été obtenu directement à partir des données expérimentales [11]. 


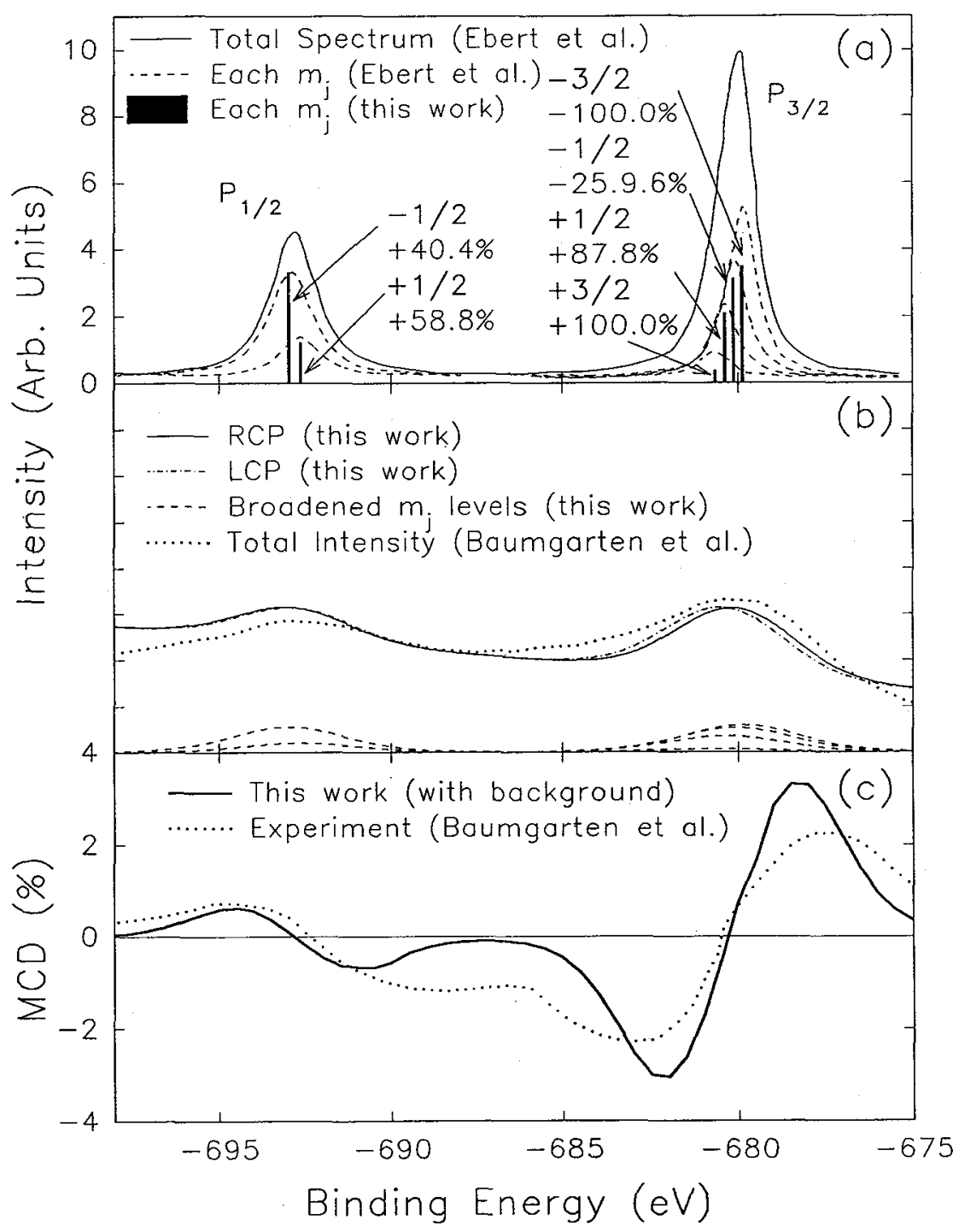

Figure 3. Comparaison de nos résultats théoriques avec ceux calculés par Ebert et al. [13], ainsi qu'avec les résultats expérimentaux de Baumgarten et al. [11], pour la photoémission du niveau Fe $2 \mathrm{p}$ de $\mathrm{Fe}(110)$, utilisant la lumière polarisée circulairement. (a) : Les résultats d'Ebert et al. [13] pour la polarisation circulaire gauche sont présentés en courbes en tirets (contributions séparées des niveaux individuels) et en corbe continue (somme des niveaux individuels). Les barres verticales représentent nos intensités simulées pour chaque niveau mj. (b) : Les courbes en tirets donnent les spectres élargis à partir des barres verticales de la figure (a). Leur somme pour la lumière à polarisation circulaire gauche est représentée par la courbe supérieure en tirets. La courbe continue donne le spectre correspondant total pour la lumière à polarisation circulaire droite. Un fond continu linéaire dérivé de l'expérience a été ajouté aux deux spectres totaux. La courbe en pointillés donne le spectre expérimental de Baumgarten et al. [11] pour la lumière polarisée linéairement. (c) : Les courbes DMC simulée (courbe continue) et expérimentale (courbe en pointillés). 
Finalement, la figure 3(c) compare les DMC simulé et expérimental pour ce système ; il est à noter que les effets expérimentaux sont très petits ici, de quelques \% seulement. L'accord entre ces deux courbes est excellent, surtout compte tenu du fait que nous avons utilisé un groupe ne comprenant que 5 atomes pour représenter la surface entière de $\mathrm{Fe}(110)$.

La seconde expérience de PS sur échantillon de Fe a été réalisée par Van Campen et al. [12], en utilisant du rayonnement non-polarisé de la ligne $\mathrm{Al} \mathrm{Ka}(1486,7 \mathrm{eV})$, pour un film de $\mathrm{Fe}$ magnétisé mais polycristallin. Dans cette expérience la polarisation en spin des photoélectrons a été mesurée directement pour les deux niveaux Fe $2 \mathrm{p} 1 / 2$ et $2 \mathrm{p} 3 / 2$. Nous présentons dans la partie supérieure de la figure 4 des spectres calculés et expérimentaux résolus en spin pour le niveau $\mathrm{Fe} 2 \mathrm{p} 3 / 2$. Les symboles triangulaires pointant vers le haut et vers le bas représentent les spectres expérimentaux avec spin $+1 / 2$ et spin $-1 / 2$ après soustraction d'un fond continu linéaire des données expérimentales d'origine. Les calculs qui sont représentés avec des courbes continues et en tirets ont été réalisés pour un atome de $\mathrm{Fe}$ isolé, qui joue le rôle d'un atome représentatif d'une surface polycristalline de $\mathrm{Fe}$; on s'attend à ce que les effets de diffraction des photoélectrons soient réduits en moyenne en raison du caractère polycristallin de l'échantillon. Les barres verticales représentent les intensités calculées pour chaque niveau mj. Leur séparation est identique à celle utilisée par Ebert et al. [13] pour expliquer les données de DMC expérimentales. Les courbes continues et en tirets donnent de nouveau la somme de nos intensités calculées pour les différents niveaux mj élargis. La hauteur des spectres calculés pour les électrons avec spin $+1 / 2$ (courbe continue) a été ajustée pour égaler celle de l'expérience. Ceci produit un accord très satisfaisant entre l'expérience et la simulation pour l'intensité de spin $+1 / 2$. Pour l'intensité de spin $-1 / 2$ nous prédisons bien la largeur du pic, mais la hauteur du pic ne concorde pas. Ceci est dû, au moins en partie, au fait que nous n'avons pas inclus de séparation des multiplets dans notre simulation, étant donné que les états finaux à multiplicité plus élevée sont toujours plus intenses pour les énergies cinétiques inférieures.

Nous montrons aussi, dans la partie inférieure de la figure 4, des polarisations en spin calculées et expérimentales. Il est encourageant de constater que les deux courbes ont la même forme générale. Le seul désaccord notable est que la polarisation en spin calculée n'a à peu près que la moitié de la valeur expérimentale dans la région des intensités maximales $(706-708 \mathrm{eV})$. Ceci est dû en grande partie à notre sous-estimation de la hauteur du pic pour spin $-1 / 2$.

\section{CONCLUSIONS}

Les exemples discutés dans cet article illustrent le bon accord qui peut être obtenu maintenant entre, d'une part, un formalisme de multi-diffraction de photoélectrons, y compris les cas du dichroïsme circulaire et les effets magnétiques, et, d'autre part, des expériences réalisées pour une grande variété de systèmes, allant d'atomes isolés de gaz rare aux surfaces magnétiques de monocristal.

Il s'avère que le dichroïsme circulaire est un effet très général, qui n'exige pas une structure chirale dans le sens classique du terme (c'est à dire une asymétrie structurelle), ni la présence d'ordre magnétique. Le dichrö̈sme existe déjà pour un système non-chiral, c'est à dire pour un sytème dont la géométrie structurelle a des symétries. Il suffit que la géométrie de la diffraction soit asymétrique par rapport à la surface ou à un groupe d'atomes; ceci arrive, par exemple, quand la direction d'incidence du photon et/ou la direction d'émission des électrons brisent la symétrie de miroir du système.

Il est important d'observer que le dichroïsme magnétique, qui est généralement faible, s'ajoute au dichroïsme structurel, qui peut être beaucoup plus fort. De plus le dichroïsme structurel est généralement hautement anisotrope. Il est donc relativement difficile d'extraire la composante magnétique du dichroïsme mesuré. Il faut done choisir une géométrie de diffraction correcte, et la choisir avec précision, pour pouvoir extraire du dichroïsme magnétique des détails sur l'état magnétique de l'échantillon. 


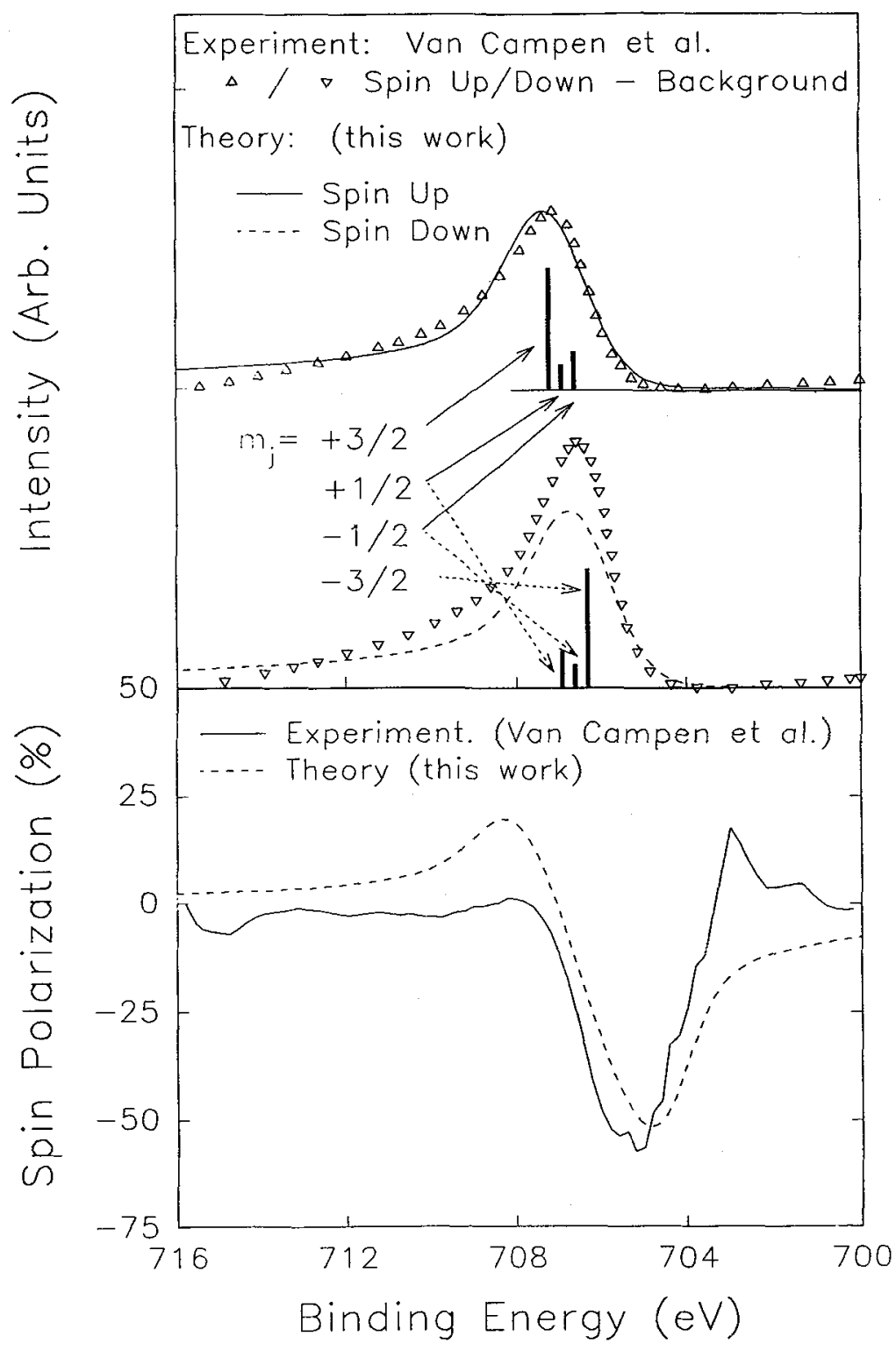

Figure 4. Spectres de photoélectrons résolus en spin du niveau Fe 2p $p_{3 / 2}$ pour le Fe polycristallin, obtenus par Van Campen et al. [12] (courbes en triangles dans la partie supérieure de la figure), et la PS dérivée de ceux-ci (partie inférieure de la figure). Nos résultats théoriques sont également présentés. Un fond continu linéaire a été soustrait des intensités expérimentales pour permettre la comparaison directe avec la théorie. Les spectres pour spin +1/2 ("spin up") ont été déplacés vers le haut pour offrir plus de clarté. 
Finalement, pour tout atome, l'excitation d'électrons à partir de niveaux séparés par l'interaction spinorbite, à l'aide de lumière à polarisation circulaire, produit une forte polarisation en spin. De plus, cette émission mène à une distribution angulaire de la polarisation fortement anisotrope. Cette anisotropie devrait être utile dans l'exploration de l'ordre magnétique à courte distance par SPEXAFS ou SPPD ; d'autre part, il faut aussi en tenir compte dans l'interprétation théorique de telles expériences, sans quoi les interprétations pourraient être sérieusement faussées.

\section{Remerciements}

Ce travail a été soutenu en partie par l'"Office of Naval Research" (Contrats Nos. N00014-90-5-1457 et N00014-94-1-0162), par le "Director, Office of Energy Research, Office of Basic Energy Sciences, Materials Sciences Division of the U.S. Department of Energy" (Contrat No. DE-AC03-76SF00098), par le "San Diego Supercomputer Center", et par le "National Energy Research Supercomputing Center" du "U.S. Department of Energy". Nous remercions K Starke de nous avoir fourni des données expérimentales sur $W(110)$ avant leur publication, et de nombreuses suggestions très utiles. Nous remercions également A.P. Kaduwela, H. Xiao et W Schattke pour leurs contributions à ce travail.

\section{Références}

1. Rehr, J.J., Albers, R.C., Phys. Rev. B41 (1990) 81139.

2. Kaduwela, A.P., Friedman, D.J., Fadley, C.S., J. Electron Spectrosc. Relat. Phenom. 57 (1991) 223.

3. Kaduwela, A.P., Xiao, H., Thevuthasan, S., Van Hove, M.A., Fadley, C.S.. Phys. Rev. B52 (1995) 14927.

4. Bansmann, J., Ostertag, Ch., Schönhense, G., Fegel, F., Westphal, C., Getzlaff, M., Schäfers, F., Petersen, H., Phys. Rev. B46 (1992) 13496.

5. Westphal, C., Kaduwela, A.P., Fadley, C.S., Van Hove, M.A., Phys. Rev. B50 (1994) 6203.

6. V. McKoy et J.A. Stephens (communication privée).

7. Daimon, H., Nakatani, T., Imada, S., Suga, S., Kagoshima, Y., Miyahara, T., Jpn. J. Appl. Phys. 32 (1993) L1480; et H. Daimon (communication privée).

8. Heckenkamp, Ch., Schäfers, F., Schönhense, G., Heinzmann, U., Phys. Rev. Lett. 52 (1984) 421.

9. Starke, K, Kaduwela, A.P., Liu, Y., Johnson, P.D., Van Hove, M.A., Fadley, C.S., Chakarian, V., Chaban, E.E., Meigs, G., Chen, C.T., Phys. Rev. B53 (1996) 10544.

10. Cherepkov, N.A., Adv. At. Mol. Phys. 19 (1983) 395; Phys. Lett. A40 (1972) 119.

11. Baumgarten, L., Schneider, C.M., Petersen, H., Schäfers, F., Kirschner, J., Phys. Rev. Lett. 65 (1990) 492.

12. Van Campen, D.G., Pouliot, R.J., Klebanoff, L.E., Phys. Rev. B48 (1993) 17533.

13. Ebert, H., Baumgarten, L., Schneider, C.M., Kirschner, J., Phys. Rev. B44 (1991) 4406. 